\title{
Synthesis and Photoluminescence Properties of
}

\section{$\mathrm{MoS}_{2} /$ Graphene Hetero-Structure by Liquid Phase}

\section{Exfoliation}

Kandhasamy Durai Murugan*a ${ }^{\mathrm{a}}$, Paulpandian Muthu Mareeswaran ${ }^{\mathrm{b}}$, Chellappan Selvaraju ${ }^{\mathrm{c}}$ and Namasivayam Dhenadhayalan ${ }^{\mathrm{d}}$

${ }^{\mathrm{a}}$ Department of Bioelectronics and Biosensors, Alagappa University, Karaikudi

Tamilnadu, INDIA-630003

${ }^{\mathrm{b}}$ Department of Industrial Chemistry, Alagappa University, Karaikudi

Tamilnadu, INDIA-630003

${ }^{\mathrm{c}}$ National Centre for Ultrafast Processes, University of Madras,

Taramani Campus, Chennai - 600 113. INDIA

${ }^{\mathrm{d}}$ Department of Chemistry, National Taiwan University and Institute of Atomic and Molecular

Sciences, Academia Sinica, Taipei 10617, Taiwan

*Corresponding Auther : kdmurugan@gmail.com (Kandhasamy Durai Murugan) 


\section{ABSTRACT:}

Synthesis of $\mathrm{MoS}_{2} /$ Graphene hetero-structure in single stage, liquid-phase exfoliation in 7:3 isopropyl alcohol /water mixture and its consequences on the photoluminescence behavior of $\mathrm{MoS}_{2}$ have been studied. Thus synthesized hetero-structure was characterized using UV-visible, micro-Raman spectroscopy, Transmission electron microscopy(TEM), and Dynamic Light Scattering (DLS) analysis. UV-Visible and micro-Raman analysis confirm improved exfoliation to the level of monolayer hetero-structure. The photophysical properties of the hetero-structure were analyzed using steady state and time resolved luminescence techniques. An enhanced photoluminescence observed in the case of hetero-structure probably due to increase in the defect sites or reduction in the rate of non-radiative decay upon formation of sandwiched heterostructure. Applications of this hetero-structure for fluorescence live cell imaging carried out and the hetero-structure demonstrated a better luminescence contrast compared to its individual counterpart $\mathrm{MoS}_{2}$ in phosphate buffered saline (PBS).

\section{KEYWORDS:}

Hetero-structure, Photoluminescence, Liquid Phase Exfoliation, Micro-Raman, Cell imaging

\section{INTRODUCTION:}

The electronic and optical properties of atomically thin, two-dimensional layered Graphene and transition metal dichalcogenides (TMDCs) have attracted significant interest due their dependence on layer thickness and size. ${ }^{1-6}$ The presence of unsaturated d-orbitals and chemically active edge sites makes them as semiconductors, semimetals, ferromagnets, and even superconductors. ${ }^{2}$ Specifically, the inherent properties of 2-dimensional (2D) materials are 
altered with a decrease of layer numbers to a single layer or a few layers, making them promising candidates in various fields of science. ${ }^{5,7,8}$ Moreover, these $2 \mathrm{D}$ materials show enhanced bio-sensing and catalytic applications owing to their large surface area. ${ }^{6,9}$ In particular, Graphene is a widely known 2D material, extensively used in biosensors, batteries, photovoltaics and super capacitor applications due to its high electron mobility, thermal conductivity and large surface area. ${ }^{10}$ Unlike graphene, $\mathrm{MoS}_{2}$ is a direct band-gap semi-conductor with tunable electronic properties as a function of layer thickness and size. ${ }^{8,11,12} \mathrm{MoS}_{2}$ shows potential applications in photo-catalysis, energy storage, bio-imaging and biosensor applications. ${ }^{4,9,13,14}$ Lateral and vertical hetero-structures of graphene and TMDC's attracted much interest due to their improved performance and stability in various fields ranging from energy conversion to biomedical applications. ${ }^{15-19}$ Characteristic, high quality hetero-interface strongly affects the built in electric field and hence the charge transfer as well as dissociation of excitons. This results in the manipulation of the optical and electronic properties of 2D materials with improved performance towards specific applications in catalysis, sensing and optoelectronic devices..$^{20-25}$

Various methods have been proposed for the synthesis of high quality hetero-structures such as direct transfer of mechanically exfoliated materials, chemical vapour deposition, van-der Walls epitaxy and in-situ catalytic growth using pre-synthesized precursor materials. ${ }^{26-29}$ Large-scale synthesis of $\mathrm{MoS}_{2} / \mathrm{Gr}$ composite by hydrothermal method using Mo and $\mathrm{S}$ precursors in the presence of exfoliated graphene for electrocatalytic water splitting was reported by Ayushi Pandey et. al. ${ }^{30}$ Also, there are several reports on the large-scale synthesis of composite like materials of Graphene with other 2D materials. ${ }^{2,31,32}$ However, synthesizing vertical/lateral stacks of these 2D hetero-structures with controlled size and purity in large scale is challenging and reported very rarely in the literature. ${ }^{29,33,34}$ 
Liquid phase exfoliation (LPE) using N-Methylpyrrolidone (NMP) and other organic solvents shown to produce various monolayer materials including Graphene, $\mathrm{hBN}, \mathrm{MoS}_{2}$, WS, etc. ${ }^{35-38}$ Moreover, LPE using water and other low boiling organic solvents can efficiently produce defect free nano-sheets of 2D materials including monolayers in suspension in a wet chemical method followed by ultrasonication and centrifugation. Recently, it is reported that the liquid phase exfoliation (LPE) effectively producing dielectric nanosheets by probing and matching the surface tension components of comparatively low boiling solvents. ${ }^{39,40}$ Monolayer enriched, luminescent nanosheets of $\mathrm{MoS}_{2}$ and $\mathrm{WS}_{2}$ have been synthesized in a single stage liquid phase exfoliation with varying percentage of water:iso-propanol mixture and 3:7 combination exhibit an enhanced exfoliation for these dichalcogenides. ${ }^{41,42}$

Here, we have attempted a scalable, single stage synthesis of $\mathrm{MoS}_{2} /$ Graphene hetero-structure in water/isopropyl alcohol solvent mixture. Thus, the obtained material was characterized by spectroscopic techniques such as UV-Visible, micro-Raman, the structural aspects were probed by TEM with SEAD pattern. The luminescence properties were investigated by steady state and time resolved photoluminescence measurements. The applicability of this material towards live cell imaging was studied and a better performance is observed for the hetero structure.

\section{MATERIALS AND METHODS:}

Graphene and $\mathrm{MoS}_{2}$ crystals used in this work were purchased from 2D semiconductors, USA. All other reagents were of analytical grade and used as received. Millipore water was used throughout the study. The individually exfoliated materials were prepared following the methods described earlier. ${ }^{41}$ The hetero-structure was synthesized by slightly modifying the above protocol (Figure-1). Briefly, $5 \mathrm{mg}$ of each Graphene and $\mathrm{MoS}_{2}$ was taken in a mortar and ground into powder. Then, the contents transferred to a glass vial and sonicated for 1 hour in $3: 7 \mathrm{v} / \mathrm{v}$ 
water/IPA mixture. The resultant suspension was centrifuged at 2000 RPM for 10 minutes and the supernatant used for further characterization and studies.

Absorption spectra for the suspensions at room temperature recorded on Shimadzu 2401 UVvisible spectrophotometer with a spectral range of 250-1100nm. Photoluminescence emission and excitation spectra were recorded on Horiba-Jobin Yvon FluoroMax-4P spectrofluorimeter equipped with Xe lamp for excitation and R928P photon counting module for the detection of light emitted from the sample. The emission from the direct band gap excitation was monitored using $5 \mathrm{~mW}, 532 \mathrm{~nm} \mathrm{CW}$ laser as the excitation source and an USB spectrometer (RI Instruments \& Innovation India). Photoluminescence decay were monitored by TCSPC single photon counting technique (Model 5000U, IBH, UK) using $375 \mathrm{~nm}$, picoseconds pulse LED as the excitation source and MCP-PMT (Hamamatsu R3809U) was used for detection of photons emitted after passing through a monochromator. The decay was fitted with HORIBA Scientific decay analysis software DAS6 to a bi/tri-exponential behavior.

Micro-Raman analysis at room temperature carried on individual sheets drop casted on a precleaned borosilicate cover slip. The spectrographs recorded using a BLZE-100 HR EMCCD camera (Princeton instruments) equipped with Acton SP2500 monochromator and a confocal microscope. A $532 \mathrm{~nm}$ continuous wave, tunable power laser used for the excitation thorough $60 \mathrm{x}$ objective. Lateral size and zeta potential of the exfoliated samples were recorded using Horiba Nanopartica SZ-100 instrument by Dynamic Light Scattering and Zeta Potential measurement respectively. Transmission Electron Microscopic images with Small Angle Electron Diffraction (SEAD) recorded for the samples coated on copper grid and dried at vacuum using JEOL-2100+ 
High Resolution Transmission Electron Microscope. Confocal fluorescence images were acquired with a Zeiss LSM 710 under the excitation wavelength of $405 \mathrm{~nm}$.

\section{In vitro Fluorescence Bioimaging}

HeLa cells were obtained from American Type Culture Collection and were cultured in RPMI1640 medium supplemented with FBS (10\%), antibiotic-antimycotic solution (1\%), L-glutamine (2 mM), and non-essential amino acids (1\%). The live cells were maintained at $37{ }^{\circ} \mathrm{C}$ and $5 \%$ $\mathrm{CO}_{2}$ in a humidified chamber. HeLa cells was incubated with $\mathrm{MoS}_{2}(50 \mu \mathrm{L})$ for 30 min to equilibrate the experimental condition. After incubation, the cells were washed with PBS buffer to remove extracellular $\mathrm{MoS}_{2}$ prior to recording images. The fluorescence image of $\mathrm{MoS}_{2}$ and $\mathrm{MoS}_{2} /$ Graphene hetero-structure incubated cells was recorded at the excitation wavelength of $405 \mathrm{~nm}$ with confocal fluorescence microscopy.

\section{RESULTS AND DISCUSSION:}

UV-Visible and Micro-Raman Spectroscopic Analysis:

The UV-Visible absorption spectra recorded for the $\mathrm{MoS}_{2} / \mathrm{GR}$ heterostructure and its individual components exhibit the characteristic absorption bands of these materials. Absorption spectrum (Figure-2A, red) of exfoliated Graphene in IPA/ $\mathrm{H}_{2} \mathrm{O}$ mixture, which shows a peak around $270 \mathrm{~nm}$ and tailing until the end of the measured spectral range. This reveals that the graphene sample has both thin-layered graphene as well as thick multilayered ones. The signature absorption maxima due to exciton states of $\mathrm{MoS}_{2}$ was observed in the liquid phase exfoliated $\mathrm{MoS}_{2}$ and $\mathrm{MoS}_{2} / \mathrm{GR}$ heterostructure at $430 \mathrm{~nm}, 607 \mathrm{~nm}$ and $665 \mathrm{~nm}$ respectively and they are assigned to $\mathrm{C}, \mathrm{B}$ and $\mathrm{A}$ exciton states of Molybdinum disulphide. ${ }^{43} \mathrm{~A}$ detailed investigation on the effect of exfoliated sheet thickness and size on the absorption maximum of $\mathrm{MoS}_{2}$ has been documented before. ${ }^{5}$ The observed 'A' exciton maximum at $665 \mathrm{~nm}$ in both $\mathrm{MoS}_{2}$ and its heterostructure clearly indicates that the obtained suspension has mostly thin layers 
of 1-3 sheet thickness. The ratio between the normalised absorption low at $\sim 340$ and B excioton maximum at $607 \mathrm{~nm}\left(\mathrm{OD}_{\mathrm{B}} / \mathrm{OD}_{340}\right)$ gives us the lateral size as well as the concentration of the particles in the suspension. ${ }^{5}$ An estimated ratio of 0.38 and 0.34 indicating that the lateral size of $\mathrm{MoS}_{2}$ and $\mathrm{MoS}_{2} / \mathrm{GR}$ dispersions is about $200 \mathrm{~nm}$ respectively. These observations further confirmed by particle size measurement using dynamic light scattering.

The particles dried and re-suspended in MilliQ water used for dynamic light scattering measurements and the profile of lateral size is shown in Figure-2B. Mean lateral size of the particles was found to be $243 \pm 72 \mathrm{~nm}$ and $261 \pm 93 \mathrm{~nm}$ for $\mathrm{MoS}_{2}$ and $\mathrm{MoS}_{2} /$ Graphene heterostructure respectively. This is again consistent with the estimated lateral size by using UVVisible spectra of the sample as mentioned in the previous section. The lateral size of the particles depends on the nature exfoliating medium and ultrasonication time. ${ }^{35}$ The lateral size observed is comparable to the previous report with larger polydispersity index for $\mathrm{MoS}_{2}$ alone under identical condition. ${ }^{41}$ Moreover, the synthesized hetero-structure reveals an increase in lateral size and polydispersity index. This may attributed to formation heterostructure with varying dimension and thickness. $\mathrm{MoS}_{2}$ and $\mathrm{MoS}_{2} / \mathrm{GR}$ hetero-structure shows a zeta potential value of $-59 \mathrm{mV}$ and $-62 \mathrm{mV}$ respectively. This indicates that the surface of the exfoliated materials are negatively charged. Zeta potential of the exfoliated nano sheets of $\mathrm{MoS}_{2}$ and graphene are known to be dependent on the $\mathrm{pH}$ of the solution. ${ }^{44,45}$ Both of them show a wide range of zeta potential between $+10 \mathrm{mV}$ to $-60 \mathrm{mV}$ as a function of $\mathrm{pH}$ of the solution. A value of around $-50 \mathrm{mV}$ was reported under neutral $\mathrm{pH}$. In the present case, a slightly higher value of -63 $\mathrm{mV}$ was observed for the hetero-structure. This shows that the nature of charge density does not change upon the formation hetero-structure. 
Raman spectrum of the two dimensional materials provides wealth of information regarding the thickness, defects and more specifically the nature of interaction between layers in the case of heterostructures. Raman Spectrum of liquid phase exfoliated Graphene exhibits peaks at 1347, 1575,2444 and $2701 \mathrm{~cm}^{-1}$ and assignable to D, G, D* and 2D bands respectively (Figure-3A). An intense D band observed in the Raman signal indicating increase in defect sites leading to more number of sp $^{3}$-hybridized carbon. ${ }^{10}$ The hetero-structure exhibits Raman features at 1340, 1571, 2434 and $2671 \mathrm{~cm}^{-1}$. The peak positions are due to the same vibronic bands of graphene mentioned above with the shift in the peak positions towards lower wave number. The shift may be due to formation of hetero-structure, which in turn affect the out of plane vibrations. The integrated intensity ratio $I_{D} / I_{G}$ for the $D$ band and $G$ band is widely used for characterizing the defect quantity in graphitic materials. The estimated values of $\mathrm{I}_{\mathrm{D}} / \mathrm{I}_{\mathrm{G}}$ ratio was 0.1970 and 0.3946 for exfoliated graphene and $\mathrm{MoS}_{2} /$ Graphene hetero-structure respectively. The quantitative amount of defects in the exfoliated Graphene was estimated using Tuinstra-Koenig relation as follows $^{46,47}$

$$
L_{a}=\left(2.4 \times 10^{-10}\right) \lambda^{4}\left(\mathrm{I}_{D} / I_{G}\right)^{-1} \quad \rightarrow
$$

Where $L_{a}$ is the in-plane crystallite size in nanometer and $\lambda$ is the wavelength of the excitation source ( $532 \mathrm{~nm}$ in the present measurement). The estimated in-plane crystallite sizes were 97 and $48 \mathrm{~nm}$ for the exfoliated Graphene and $\mathrm{MoS}_{2} /$ Graphene hetero-structure respectively. The translational symmetry of the Graphene sheets was directly related to the presence of defects and structural disorders. ${ }^{48}$ An increase in defects and/or disorder in the structure lead to reduced inplane crystallite size. The density of defects calculated from the in-plane crystallite size using the following relation 


$$
n_{D}\left(\mathrm{~cm}^{-2}\right)=10^{14} / \pi L_{a}^{2} \quad \rightarrow
$$

Where $\mathrm{n}_{\mathrm{D}}$ is the defect density and estimated to be $0.33 \times 10^{10}$ and $1.34 \times 10^{10} \mathrm{~cm}^{-2}$ for the exfoliated graphene and $\mathrm{MoS}_{2} /$ Graphene hetero-structure. The decrease in the size of the in plane crystallites and higher defect density upon the formation of hetero-structure may be attributed to the effective exfoliation under the chosen solvent mixture and/or partial oxidation of Graphene during ultrasonic treatment. The width of ' $\mathrm{G}$ ' peak is also implies the nature of defects and presence of other impurities. In the present case, the observed width of $\sim 15 \mathrm{~cm}^{-1}$ for both LPE exfoliated Graphene and $\mathrm{MoS}_{2} /$ Graphene hetero-structure implies that the material is almost equal to mechanically exfoliated Graphene in purity. Such quality production of few layer hetero-structures attributed to the usage of low boiling and environmentally benign solvent mixture during the exfoliation.

Raman spectra shown in Figure -3A of $\mathrm{MoS}_{2}$ and $\mathrm{MoS}_{2} /$ Graphene hetero-structure have a peak at $\sim 400 \mathrm{~cm}^{-1}$ accompanied by a shoulder. In order to resolve the spectral signs of $\mathrm{MoS}_{2}$ a narrow scan was carried out (Figure-3B) which clearly reveals peaks at 383.6 and $404.8 \mathrm{~cm}^{-1}$ with a peak separation of $21.2 \mathrm{~cm}^{-1}$. Lattice vibrations and the intralayer bonding in stacked fewlayer crystallites perturbed by weak van der Waals interlayer interactions. $\mathrm{E}_{2 \mathrm{~g}}$ mode assigned to opposite vibration of S atoms with respect to the Mo atom and the out-of-plane vibration of only $\mathrm{S}$ atoms in opposite directions yields $\mathrm{A}_{1 \mathrm{~g}}$ mode. ${ }^{49}$ Layer thickness dependant frequency shift of both modes have been examined and the frequency of $\mathrm{E}_{2 \mathrm{~g}}$ peak decreases while that of the $\mathrm{A}_{1 \mathrm{~g}}$ peak increases with increasing layer thickness. In addition, the peak separation and the intensity of the peaks $E_{2 g} \& A_{1 g}$ increases with the increase in the number of layers. ${ }^{11}$ The liquid exfoliated $\mathrm{MoS}_{2}$ sample prepared by the present mixed solvent method have sheet with the thickness of 2-3 layers as evident from the frequencies of the $A_{1 g}$ and $E_{2 g}$ modes as well as the 
separation frequency of the two peaks. However, a shift in the peak position compared to mechanically exfoliated sample may be attributed to the nature of the solvent/material interaction or partial oxidation of $\mathrm{MoS}_{2}$ nanosheets. The peak frequencies of both $\mathrm{E}_{2 \mathrm{~g}}$ and $\mathrm{A}_{1 \mathrm{~g}}$ shows a shift towards lower wave-number in the presence of Graphene which ascribed to constrained out of plane vibrations due to the formation hetero-structure effectively. Also, it is observed in every particle or position monitored during Raman measurement shows the features of both $\mathrm{MoS}_{2}$ and Graphene. This is a clear indication of the absence of free particles of both $\mathrm{MoS}_{2}$ and Graphene in the synthesized hetero-structure. At present, the nature of interaction dominating the formation hetero-structure is not clear. However, the balance between weak hydrogen bonding, hydrophobic and hydrophilic interaction might be the driving force for the effective formation of hetero-structure during the liquid phase exfoliation.

Structural Analysis of the Heterostructure:

The size and lattice alignment of the $\mathrm{MoS}_{2} / \mathrm{GR}$ hetero-structure were analyzed by transmission electron microscopy and small angle electron diffraction as shown in Figure-4. A typical hexagonal diffraction pattern was seen in both $\mathrm{MoS}_{2}$ and Graphene SAED pattern with lattice spacing $0.67 \mathrm{~nm}$ and $0.35 \mathrm{~nm}$ respectively. ${ }^{50}$ The observed lattice spacing confirmed that the sonication assisted liquid phase exfoliation did not alter the interlayer distance as seen in some of the previous studies. The vertical stacking of synthesized hetero-structure is also evident from the high-resolution image shown in Figure-4f. Vertical hetero-structures of TMDCs with graphene have been achieved with controlled chemical vapor deposition and mechanical exfoliation followed by transfer. ${ }^{51,52}$ However, this is the first time vertical hetero-structures of $\mathrm{MoS}_{2} /$ Graphene was synthesized by a single stage, scalable liquid phase exfoliation. Moreover, the position of the SAED pattern reveals that the angle of lattice orientation between Graphene 
and $\mathrm{MoS}_{2}$ might be either $0^{\circ}$ or $60^{\circ}$ in many cases, as we did not notice any other orientation angle in the SAED pattern. The characterizations carried out above unambiguously confirmed that the mixed solvent liquid phase exfoliation adapted to synthesize hetero-structures of $\mathrm{MoS}_{2} /$ Graphene effectively worked out and the strategy is scalable for large-scale preparation of the hetero-structures with the advantage of combined/modulated optical and electrical properties of both.

The Photoluminescence behavior of $\mathrm{MoS}_{2}$ in the suspension and its heterostructure with Graphene were evaluated by steady state and time resolved measurements. $\mathrm{MoS}_{2}$ is a direct band semiconductor and the band-gap emission occurs at $1.9 \mathrm{eV}(\sim 650 \mathrm{~nm})$ due to the interband transition of $\mathrm{K}$ point. ${ }^{42}$ This interband photoluminescence observed at $688 \mathrm{~nm}$ for $\mathrm{MoS}_{2}$ on excitation using $532 \mathrm{~nm}$ in the suspension (Figure-5A). Mechanically exfoliated $\mathrm{MoS}_{2}$ monolayer exhibit strong photoluminescence with a peak at 650-690 nm and shoulder around $610 \mathrm{~nm}$ due to A and $\mathrm{B}$ excitonic bands respectively. The photoluminescence peak maximum of monolayer as well as few layer shifts on different substrates such as Sapphire, $\mathrm{SiO}_{2}, \mathrm{Si}$ and fused Silica. ${ }^{53}$ Moreover the nature of impurities also play critical role on the photoluminescence intensity and peak position. Chemically exfoliated $\mathrm{MoS}_{2}$ exhibit metallic behavior due to Lithium intercalation under normal conditions and upon mild annealing results in the restoration on intrinsic photoluminescence of thin film. ${ }^{42}$ In the present case, the observed photoluminescence slightly shifted to red-region, which may be due to solvent-solute interaction in the suspended $\mathrm{MoS}_{2}$. However, the $\mathrm{MoS}_{2} /$ Graphene hetero-structure shows peak maximum at $695 \mathrm{~nm}$ that shows a further red shift compared $\mathrm{MoS}_{2}$ alone in the solvent system. Further, it is observed that the photoluminescence intensity increased twice that of $\mathrm{MoS}_{2}$ alone. This could be explained by the fact that interlayer coupling between $\mathrm{MoS}_{2}$ and Graphene results in increase in 
the layer thickness. The photoluminescence intensity as well as the peak position depends on the layer thickness and a red shift in the emission intensity is observed upon increasing the layer thickness. ${ }^{42}$ On the other hand the increase in the PL intensity may be attributed to the close packing of $\mathrm{MoS}_{2}$ between the Graphene layers might have reduce the rate of non-radiative decay. This is further ascertained by time resolved PL decay analysis as shown in Figure-5B and table1. The decay obeys bi-exponential function with a prominent shorter decay time of $0.2 \mathrm{~ns}(95 \%)$ and a longer decay component of $4.0 \mathrm{~ns}(5 \%)$. The average decay time estimated to be $0.4 \mathrm{~ns}$. $\mathrm{MoS}_{2} /$ Graphene heterostructure also follows bi-exponential decay with decay times of $4.10 \mathrm{~ns}$ $(10 \%) \& 0.25$ ns $(90 \%)$ and the average lifetime was found to be 0.64 ns in the suspension. An average lifetime of 410 ps was reported by Mohamed et. al for the monolayer $\mathrm{MoS}_{2}$ and the observed lifetime of 420 ps matches with their reports for the $\mathrm{MoS}_{2}$ suspension. ${ }^{54}$ Moreover an enhancement in the decay time upon the formation of heterostructure is consistent with the enhancement of luminescence intensity. This is again validating that upon the formation of heterostructure the rate of non-radiative decay channels are suppressed.

Earlier, it has been observed that the suspension of $\mathrm{MoS}_{2}$ in solution exhibits strong luminescence in the blue region due to defect sites or size quantization in addition to band edge emission. ${ }^{55-57}$ The effect of heterostructure on the photoluminescence properties of $\mathrm{MoS}_{2}$ due to defect sites have also been explored. Figure $5 \mathrm{c} \& 5 \mathrm{~d}$ show the steady state and time resolved photoluminescence of $\mathrm{MoS}_{2}$ and $\mathrm{MoS}_{2}$ /Graphene heterostructure. Maximum photoluminescence intensity was observed upon excitation at $360 \mathrm{~nm}$ with a peak at $430 \mathrm{~nm}$ and respective excitation spectrum is also shown in the Figure-5c. $\mathrm{MoS}_{2}$ sheets exhibits an excitation dependant emission in the blue region and attributed to the inhomogeneous size distribution and the presence of multiple trap states. ${ }^{38,55}$ Interestingly, more than 20 fold enhancement in the emission intensity 
was observed for the heterostructure as seen in the Figure-5c. This may due to the combined effect of increase in the number of defect sites and the reduction in the non-radiative rate in the heterostructure. This is further ascertained by measuring the time resolved decay profile as shown in Figure-5d. The decay profile display a bi-exponential decay with decay times of $5.3 \mathrm{~ns}$ $(67 \%)$ and $1.95 \mathrm{~ns}(33 \%)$ for $\mathrm{MoS}_{2}$. The average decay times were estimated to be $4.23 \mathrm{~ns}$ and 4.25 ns for $\mathrm{MoS}_{2}$ and $\mathrm{MoS}_{2} /$ Graphene heterostructure respectively. The nearly identical decay time for both and an enhancement in the PL intensity may be correlated to the increase in defect sites upon the heterostructure formation.

The effect of $\mathrm{MoS}_{2}$ on normal and cancer cell lines have been studied earlier and the results indicate that $\mathrm{MoS}_{2}$ adhesion lead to cell death in cancerous cell lines and unaffected the normal cell lines after 24 hours of incubation. ${ }^{14}$ The uptake of $\mathrm{MoS}_{2}$ and its localization inside the cell probed by transient absorption and Raman scattering in a custom built dual mode microscope using HeLa cell lines. ${ }^{58}$ However, live cell imaging using $\mathrm{MoS}_{2}$ as a luminescent probe is limited due to the poor photoluminescence of the material in biological medium. ${ }^{55,59}$ The hetero-structure obtained by the present strategy has more than 20 fold enhancement in the luminescence intensity compared to $\mathrm{MoS}_{2}$ nanosheets under identical condition might be potential candidate for live cell imaging using photoluminescence of the material. HeLa cell lines incubated with $50 \mathrm{ml}$ of the re-suspended $\mathrm{MoS}_{2} /$ Graphene heterostructure were used for the imaging as mentioned in the experimental section in biological buffer medium (Figure-6). Bright field images of the cells incubated with $50 \mu \mathrm{l}$ of the redispersed $\mathrm{MoS}_{2}$ and its hetero-structure is shown in Figure-6a\&d and it is noticed that the cells are alive upto $12 \mathrm{hrs}$ in the incubated medium. Fluorescent and merged images of the cells reveal that both the materials internalized to 
the cytoplasm of the cells. In addition, it is clearly visible that the hetero-structure has enhanced fluorescent contrast compared to $\mathrm{MoS}_{2}$ alone under identical environment.

\section{CONCLUSION:}

Hetero-structure of $\mathrm{MoS}_{2}$ with Graphene was successfully made by a single stage liquid phase exfoliation using IPA: water mixture(7:3 v/v). Thus obtained Vander-Walls heterostructure was characterized by Raman, UV-Visible, TEM and Dynamic light scattering studies. The heterostructures have 2-5 layers of $\mathrm{MoS}_{2}$ as evident from Raman and UV-Visible analysis. DLS, UV-visible and TEM analysis revealed that the lateral sizes of the material was about 200 $\mathrm{nm}$ and surface of both $\mathrm{MoS}_{2}$ and hetero-structure negatively charged with $\zeta$-potential values of $\sim-60 \mathrm{mV}$. TEM with SAED confirms the formation of vertical hetero-structure of $\mathrm{MoS}_{2} /$ Graphene with the present strategy. About 20 fold enhancement in the photoluminescence intensity attributed to the suppression of non-radiative decay pathways due to the formation of heterostructure that is also clear from higher decay times in time resolved photoluminescence measurement. The material shows potential application in live cell imaging using photoluminescence compared to $\mathrm{MoS}_{2}$ with the added advantage of Graphene in the heterostructure.

\section{ACKNOWLEDGMENT:}

The authors D. K and P. M thank the financial support from RUSA 2.0 (MHRD-India) grant sanctioned vide Letter No. F. 24-51/2014-U, Policy (TNMulti-Gen), Dept. of Edn. Govt. of India, Dt. October 09, 2018.

\section{REFERENCES}

(1) Wang, Q. H.; Kalantar-Zadeh, K.; Kis, A.; Coleman, J. N.; Strano, M. S. Electronics and Optoelectronics of Two-Dimensional Transition Metal Dichalcogenides. Nat. 
Nanotechnol. 2012, 7 (11), 699-712. https://doi.org/10.1038/nnano.2012.193.

(2) Chhowalla, M.; Shin, H. S.; Eda, G.; Li, L. J.; Loh, K. P.; Zhang, H. The Chemistry of Two-Dimensional Layered Transition Metal Dichalcogenide Nanosheets. Nat. Chem. 2013, 5 (4), 263-275. https://doi.org/10.1038/nchem.1589.

(3) Weng, M.; Li, S.; Zheng, J.; Pan, F.; Wang, L. W. Wannier Koopmans Method Calculations of 2D Material Band Gaps. J. Phys. Chem. Lett. 2018, 9 (2), 281-285. https://doi.org/10.1021/acs.jpclett.7b03041.

(4) Yadav, V.; Roy, S.; Singh, P.; Khan, Z.; Jaiswal, A. 2D MoS2-Based Nanomaterials for Therapeutic, Bioimaging, and Biosensing Applications. Small 2019, 15 (1), 1-33. https://doi.org/10.1002/smll.201803706.

(5) Backes, C.; Smith, R. J.; McEvoy, N.; Berner, N. C.; McCloskey, D.; Nerl, H. C.; O’Neill, A.; King, P. J.; Higgins, T.; Hanlon, D.; et al. Edge and Confinement Effects Allow in Situ Measurement of Size and Thickness of Liquid-Exfoliated Nanosheets. Nat. Commun. 2014, 5, 1-10. https://doi.org/10.1038/ncomms5576.

(6) Zhang, W.; Zhang, P.; Su, Z.; Wei, G. Synthesis and Sensor Applications of MoS 2 Based Nanocomposites. Nanoscale 2015, $7 \quad$ (44), $\quad$ 18364-18378. https://doi.org/10.1039/c5nr06121k.

(7) Papanai, G. S.; Sharma, I.; Kedawat, G.; Gupta, B. K. Qualitative Analysis of Mechanically Exfoliated MoS 2 Nanosheets Using Spectroscopic Probes. https://doi.org/10.1021/acs.jpcc.9b09191.

(8) Nerl, H. C.; Winther, K. T.; Hage, F. S.; Thygesen, K. S.; Houben, L.; Backes, C.; Coleman, J. N.; Ramasse, Q. M.; Nicolosi, V. Probing the Local Nature of Excitons and Plasmons in Few-Layer MoS2. npj 2D Mater. Appl. 2017, 1 (1), 1-8. 
https://doi.org/10.1038/s41699-017-0003-9.

(9) Kalantar-Zadeh, K.; Ou, J. Z. Biosensors Based on Two-Dimensional MoS2. ACS Sensors 2016, 1 (1), 5-16. https://doi.org/10.1021/acssensors.5b00142.

(10) Wu, J. Bin; Lin, M. L.; Cong, X.; Liu, H. N.; Tan, P. H. Raman Spectroscopy of Graphene-Based Materials and Its Applications in Related Devices. Chem. Soc. Rev. 2018, 47 (5), 1822-1873. https://doi.org/10.1039/c6cs00915h.

(11) Lee, C.; Yan, H.; Brus, L. E.; Heinz, T. F.; Hone, J.; Ryu, S. Anomalous Lattice Vibrations of Single- and Few-Layer MoS2. ACS Nano 2010, 4 (5), 2695-2700. https://doi.org/10.1021/nn1003937.

(12) Koitzsch, A.; Pawlik, A. S.; Habenicht, C.; Klaproth, T.; Schuster, R.; Büchner, B.; Knupfer, M. Nonlocal Dielectric Function and Nested Dark Excitons in MoS2. npj 2D Mater. Appl. 2019, 3 (1). https://doi.org/10.1038/s41699-019-0122-6.

(13) Wang, Q.; Huang, J.; Sun, H.; Ng, Y. H.; Zhang, K. Q.; Lai, Y. MoS2 Quantum Dots@TiO2 Nanotube Arrays: An Extended-Spectrum-Driven Photocatalyst for Solar Hydrogen Evolution. ChemSusChem 2018, 11 (10), 1708-1721. https://doi.org/10.1002/cssc.201800379.

(14) Kaur, J.; Singh, M.; Dell‘Aversana, C.; Benedetti, R.; Giardina, P.; Rossi, M.; Valadan, M.; Vergara, A.; Cutarelli, A.; Montone, A. M. I.; et al. Biological Interactions of Biocompatible and Water-Dispersed MoS2 Nanosheets with Bacteria and Human Cells. Sci. Rep. 2018, 8 (1), 1-15. https://doi.org/10.1038/s41598-018-34679-y.

(15) Faridi, E.; Moradi, M.; Ansari, N.; Ghasemi, A. H. B.; Afshar, A.; Mohseni, S. M. $\mathrm{Au} / \mathrm{NiFe} / \mathrm{M}(\mathrm{Au}, \mathrm{MoS} 2$, Graphene) Trilayer Magnetoplasmonics DNA-Hybridized Sensors with High Record of Sensitivity. J. Biomed. Opt. 2017, 22 (12), 1. 
https://doi.org/10.1117/1.jbo.22.12.127001.

(16) Li, X.; Guo, S.; Li, W.; Ren, X.; Su, J.; Song, Q.; Sobrido, A. J.; Wei, B. Edge-Rich MoS2

Grown on Edge-Oriented Three-Dimensional Graphene Glass for High-Performance Hydrogen Evolution. Nano Energy 2019, 57, 388-397. https://doi.org/10.1016/j.nanoen.2018.12.044.

(17) Mukherjee, S.; Maiti, R.; Katiyar, A. K.; Das, S.; Ray, S. K. Novel Colloidal MoS 2 Quantum Dot Heterojunctions on Silicon Platforms for Multifunctional Optoelectronic Devices. Sci. Rep. 2016, 6 (June). https://doi.org/10.1038/srep29016.

(18) Naz, R.; Imtiaz, M.; Liu, Q.; Yao, L.; Abbas, W.; Li, T.; Zada, I.; Yuan, Y.; Chen, W.; Gu, J. Highly Defective 1T-MoS2 Nanosheets on 3D Reduced Graphene Oxide Networks for Supercapacitors. Carbon N. Y. 2019, 152, 697-703. https://doi.org/10.1016/j.carbon.2019.06.009.

(19) Sahoo, P. K.; Memaran, S.; Nugera, F. A.; Xin, Y.; Díaz Márquez, T.; Lu, Z.; Zheng, W.; Zhigadlo, N. D.; Smirnov, D.; Balicas, L.; et al. Bilayer Lateral Heterostructures of Transition-Metal Dichalcogenides and Their Optoelectronic Response. ACS Nano 2019. https://doi.org/10.1021/acsnano.9b04957.

(20) Xiao, R.; Lan, C.; Li, Y.; Zeng, C.; He, T.; Wang, S.; Li, C.; Yin, Y.; Liu, Y. High Performance Van Der Waals Graphene-WS2-Si Heterostructure Photodetector. Adv. Mater. Interfaces 2019, 1901304, 1-7. https://doi.org/10.1002/admi.201901304.

(21) Luo, Y. K.; Xu, J.; Zhu, T.; Wu, G.; McCormick, E. J.; Zhan, W.; Neupane, M. R.; Kawakami, R. K. Opto-Valleytronic Spin Injection in Monolayer MoS2/Few-Layer Graphene Hybrid Spin Valves. Nano Lett. 2017, 17 (6), 3877-3883. https://doi.org/10.1021/acs.nanolett.7b01393. 
(22) Zheng, G.; Zou, X.; Chen, Y.; Xu, L.; Rao, W. Fano Resonance in Graphene-MoS2 Heterostructure-Based Surface Plasmon Resonance Biosensor and Its Potential Applications. Opt. Mater. (Amst). 2017, 66, 171-178. https://doi.org/10.1016/j.optmat.2017.02.001.

(23) Xu, L.; Huang, W. Q.; Hu, W.; Yang, K.; Zhou, B. X.; Pan, A.; Huang, G. F. TwoDimensional MoS2-Graphene-Based Multilayer van Der Waals Heterostructures: Enhanced Charge Transfer and Optical Absorption, and Electric-Field Tunable Dirac Point and Band Gap. Chem. Mater. 2017, 29 (13), 5504-5512. https://doi.org/10.1021/acs.chemmater.7b00281.

(24) Romanov, R. I.; Slavich, A. S.; Kozodaev, M. G.; Myakota, D. I.; Lebedinskii, Y. Y.; Novikov, S. M.; Markeev, A. M. Band Alignment in As-Transferred and Annealed Graphene/MoS2 Heterostructures. Phys. Status Solidi - Rapid Res. Lett. 2019, 1900406, 1-6. https://doi.org/10.1002/pssr.201900406.

(25) Zhou, Y.; Yang, Y.; Guo, Y.; Wang, Q.; Yan, X. Influence of Length and Interface Structure on Electron Transport Properties of Graphene-MoS2 in-Plane Heterojunction. Appl. Surf. Sci. 2019, 497 (April), 143764. https://doi.org/10.1016/j.apsusc.2019.143764.

(26) Miwa, J. A.; Dendzik, M.; Grønborg, S. S.; Bianchi, M.; Lauritsen, J. V.; Hofmann, P.; Ulstrup, S. Van Der Waals Epitaxy of Two-Dimensional $\operatorname{MoS}<\inf >2</ \operatorname{Inf}>$-Graphene Heterostructures in Ultrahigh Vacuum. ACS Nano 2015, 9 (6), 6502-6510. https://doi.org/10.1021/acsnano.5b02345.

(27) Rathi, S.; Lee, I.; Lim, D.; Wang, J.; Ochiai, Y.; Aoki, N.; Watanabe, K.; Taniguchi, T.; Lee, G. H.; Yu, Y. J.; et al. Tunable Electrical and Optical Characteristics in Monolayer Graphene and Few-Layer MoS2 Heterostructure Devices. Nano Lett. 2015, 15 (8), 5017 - 
5024. https://doi.org/10.1021/acs.nanolett.5b01030.

(28) Zhang, Y.; Shi, J.; Liu, M.; Wen, J.; Ren, X.; Zhou, X.; Ji, Q.; Ma, D.; Zhang, Y.; Jin, C.; et al. All Chemical Vapor Deposition Synthesis and Intrinsic Bandgap Observation of MoS2/Graphene Heterostructures. Adv. Mater. 2015, 27 (44), 7086-7092. https://doi.org/10.1002/adma.201503342.

(29) Fu, W.; Du, F. H.; Su, J.; Li, X. H.; Wei, X.; Ye, T. N.; Wang, K. X.; Chen, J. S. In Situ Catalytic Growth of Large-Area Multilayered Graphene/MoS 2 Heterostructures. Sci. Rep. 2014, 4 (July). https://doi.org/10.1038/srep04673.

(30) Pandey, A.; Mukherjee, A.; Chakrabarty, S.; Chanda, D.; Basu, S. Interface Engineering of an $\mathrm{RGO} / \mathrm{MoS} 2 / \mathrm{Pd} 2 \mathrm{D}$ Heterostructure for Electrocatalytic Overall Water Splitting in Alkaline Medium. ACS Appl. Mater. Interfaces 2019, 11, 42094-42103. https://doi.org/10.1021/acsami.9b13358.

(31) Han, J.; Yan, T.; Shen, J.; Shi, L.; Zhang, J.; Zhang, D. Capacitive Deionization of Saline Water by Using MoS2-Graphene Hybrid Electrodes with High Volumetric Adsorption Capacity. Environ. Sci. $\quad$ Technol. 2019, $53 \quad$ (21), $12668-12676$. https://doi.org/10.1021/acs.est.9b04274.

(32) Zhao, P.; Ni, M.; Xu, Y.; Wang, C.; Chen, C.; Zhang, X.; Li, C.; Xie, Y.; Fei, J. A Novel Ultrasensitive Electrochemical Quercetin Sensor Based on MoS2 - Carbon Nanotube @ Graphene Oxide Nanoribbons / HS-Cyclodextrin / Graphene Quantum Dots Composite Film. Sensors Actuators, B Chem. 2019, 299 (March), 126997. https://doi.org/10.1016/j.snb.2019.126997.

(33) Kim, Y.; Choi, D.; Woo, W. J.; Lee, J. B.; Ryu, G. H.; Lim, J. H.; Lee, S.; Lee, Z.; Im, S.; Ahn, J. H.; et al. Synthesis of Two-Dimensional MoS2/Graphene Heterostructure by 
Atomic Layer Deposition Using MoF6 Precursor. Appl. Surf. Sci. 2019, 494 (July), 591599. https://doi.org/10.1016/j.apsusc.2019.07.168.

(34) Hou, L.; Zhang, Q.; Tweedie, M.; Shautsova, V.; Sheng, Y.; Zhou, Y.; Huang, H.; Chen, T.; Warner, J. H. Photocurrent Direction Control and Increased Photovoltaic Effects in All-2D Ultrathin Vertical Heterostructures Using Asymmetric h-BN Tunneling Barriers. ACS Appl. Mater. Interfaces 2019, $11 \quad$ (43), 40274-40282. https://doi.org/10.1021/acsami.9b13404.

(35) Dong, L.; Lin, S.; Yang, L.; Zhang, J.; Yang, C.; Yang, D.; Lu, H. Spontaneous Exfoliation and Tailoring of MoS2 in Mixed Solvents. Chem. Commun. 2014, 50 (100), 15936-15939. https://doi.org/10.1039/c4cc07238c.

(36) Jawaid, A.; Nepal, D.; Park, K.; Jespersen, M.; Qualley, A.; Mirau, P.; Drummy, L. F.; Vaia, R. A. Mechanism for Liquid Phase Exfoliation of MoS2. Chem. Mater. 2016, 28 (1), 337-348. https://doi.org/10.1021/acs.chemmater.5b04224.

(37) Xu, Y.; Cao, H.; Xue, Y.; Li, B.; Cai, W. Liquid-Phase Exfoliation of Graphene: An Overview on Exfoliation Media, Techniques, and Challenges. Nanomaterials 2018, 8 (11). https://doi.org/10.3390/nano8110942.

(38) Wu, J. Y.; Zhang, X. Y.; Ma, X. D.; Qiu, Y. P.; Zhang, T. High Quantum-Yield Luminescent MoS2 Quantum Dots with Variable Light Emission Created via Direct Ultrasonic Exfoliation of MoS2 Nanosheets. RSC Adv. 2015, 5 (115), 95178-95182. https://doi.org/10.1039/c5ra19201c.

(39) Geim, A. K.; Grigorieva, I. V. Van Der Waals Heterostructures. Nature 2013, 499 (7459), 419-425. https://doi.org/10.1038/nature12385.

(40) Shen, J.; He, Y.; Wu, J.; Gao, C.; Keyshar, K.; Zhang, X.; Yang, Y.; Ye, M.; Vajtai, R.; 
Lou, J.; et al. Liquid Phase Exfoliation of Two-Dimensional Materials by Directly Probing and Matching Surface Tension Components. Nano Lett. 2015, 15 (8), 5449-5454. https://doi.org/10.1021/acs.nanolett.5b01842.

(41) Bernal, M. M.; Álvarez, L.; Giovanelli, E.; Arnáiz, A.; Ruiz-González, L.; Casado, S.; Granados, D.; Pizarro, A. M.; Castellanos-Gomez, A.; Pérez, E. M. Luminescent Transition Metal Dichalcogenide Nanosheets through One-Step Liquid Phase Exfoliation. 2D Mater. 2016, 3 (3). https://doi.org/10.1088/2053-1583/3/3/035014.

(42) Eda, G.; Yamaguchi, H.; Voiry, D.; Fujita, T.; Chen, M.; Chhowalla, M. Photoluminescence from Chemically Exfoliated MoS 2. Nano Lett. 2011, 11 (12), 51115116. https://doi.org/10.1021/nl201874w.

(43) Klots, A. R.; Newaz, A. K. M.; Wang, B.; Prasai, D.; Krzyzanowska, H.; Lin, J.; Caudel, D.; Ghimire, N. J.; Yan, J.; Ivanov, B. L.; et al. Probing Excitonic States in Suspended Two-Dimensional Semiconductors by Photocurrent Spectroscopy. Sci. Rep. 2014, 4 (May). https://doi.org/10.1038/srep06608.

(44) Konkena, B.; Vasudevan, S. Understanding Aqueous Dispersibility of Graphene Oxide and Reduced Graphene Oxide through p K a Measurements. J. Phys. Chem. Lett. 2012, 3 (7), 867-872. https://doi.org/10.1021/jz300236w.

(45) Yadgarov, L.; Choi, C. L.; Sedova, A.; Cohen, A.; Rosentsveig, R.; Bar-Elli, O.; Oron, D.; Dai, H.; Tenne, R. Dependence of the Absorption and Optical Surface Plasmon Scattering of MoS2 Nanoparticles on Aspect Ratio, Size, and Media. ACS Nano 2014, 8 (4), 35753583. https://doi.org/10.1021/nn5000354.

(46) Tuinstra, F.; Koenig, J. L. Raman Spectrum of Graphite. J. Chem. Phys. 1970, 53 (3), 1126-1130. https://doi.org/10.1063/1.1674108. 
(47) Cançado, L. G.; Jorio, A.; Ferreira, E. H. M.; Stavale, F.; Achete, C. A.; Capaz, R. B.; Moutinho, M. V. O.; Lombardo, A.; Kulmala, T. S.; Ferrari, A. C. Quantifying Defects in Graphene via Raman Spectroscopy at Different Excitation Energies. Nano Lett. 2011, 11 (8), 3190-3196. https://doi.org/10.1021/nl201432g.

(48) Backes, C.; , Amr M Abdelkader, Concepción Alonso, A. A.-L. et. al. Production and Processing of Graphene and Related Materials. 2D Mater 2020, 7, 022001.

(49) Wu, P. R.; Liu, Z.; Cheng, Z. L. Hydrothermal-Assisted Shearing Exfoliation for FewLayered MoS2 Nanosheets. RSC Adv. 2019, 9 (30), 17016-17024. https://doi.org/10.1039/c9ra02102g.

(50) Liao, M.; Wu, Z. W.; Du, L.; Zhang, T.; Wei, Z.; Zhu, J.; Yu, H.; Tang, J.; Gu, L.; Xing, Y.; et al. Twist Angle-Dependent Conductivities across MoS 2 /Graphene Heterojunctions. Nat. Commun. 2018, 9 (1), 1-6. https://doi.org/10.1038/s41467-01806555-w.

(51) Shi, J.; Zhou, X.; Han, G. F.; Liu, M.; Ma, D.; Sun, J.; Li, C.; Ji, Q.; Zhang, Y.; Song, X.; et al. Narrow-Gap Quantum Wires Arising from the Edges of Monolayer MoS2 Synthesized on Graphene. Adv. Mater. Interfaces 2016, 3 (17). https://doi.org/10.1002/admi.201600332.

(52) Safeer, C. K.; Ingla-Aynés, J.; Herling, F.; Garcia, J. H.; Vila, M.; Ontoso, N.; Calvo, M. R.; Roche, S.; Hueso, L. E.; Casanova, F. Room-Temperature Spin Hall Effect in Graphene/MoS2 van Der Waals Heterostructures. Nano Lett. 2019. https://doi.org/10.1021/acs.nanolett.8b04368.

(53) Mohapatra, P. K.; Deb, S.; Singh, B. P.; Vasa, P.; Dhar, S. Strictly Monolayer Large Continuous MoS2 Films on Diverse Substrates and Their Luminescence Properties. Appl. 
Phys. Lett. 2016, 108 (4). https://doi.org/10.1063/1.4940751.

(54) Mohamed, N. B.; Lim, H. E.; Wang, F.; Koirala, S.; Mouri, S.; Shinokita, K.; Miyauchi, Y.; Matsuda, K. Long Radiative Lifetimes of Excitons in Monolayer Transition-Metal Dichalcogenides MX $2(\mathrm{M}=\mathrm{Mo}, \mathrm{W} ; \mathrm{X}=\mathrm{S}$, Se). Appl. Phys. Express 2018, 11 (1), 0-4. https://doi.org/10.7567/APEX.11.015201.

(55) Zhou, K.; Zhang, Y.; Xia, Z.; Wei, W. As-Prepared MoS2 Quantum Dot as a Facile Fluorescent Probe for Long-Term Tracing of Live Cells. Nanotechnology 2016, 27 (27), 1-8. https://doi.org/10.1088/0957-4484/27/27/275101.

(56) Wang, N.; Wei, F.; Qi, Y.; Li, H.; Lu, X.; Zhao, G.; Xu, Q. Synthesis of Strongly Fluorescent Molybdenum Disulfide Nanosheets for Cell-Targeted Labeling. ACS Appl. Mater. Interfaces 2014, 6 (22), 19888-19894. https://doi.org/10.1021/am505305g.

(57) Wu, J. Y.; Lin, M. N.; Wang, L. De; Zhang, T. Photoluminescence of MoS2 Prepared by Effective Grinding-Assisted Sonication Exfoliation. J. Nanomater. 2014, 2014 (Cvd). https://doi.org/10.1155/2014/852735.

(58) Zhang, L.; Shen, S.; Liu, Z.; Ji, M. Label-Free, Quantitative Imaging of MoS 2 Nanosheets in Live Cells with Simultaneous Stimulated Raman Scattering and Transient Absorption Microscopy . Adv. Biosyst. 2017, $1 \quad 1$ (4), 1700013. https://doi.org/10.1002/adbi.201700013.

(59) Feng, W.; Chen, L.; Qin, M.; Zhou, X.; Zhang, Q.; Miao, Y.; Qiu, K.; Zhang, Y.; He, C. Flower-like PEGylated MoS2 Nanoflakes for near-Infrared Photothermal Cancer Therapy. Sci. Rep. 2015, 5, 1-13. https://doi.org/10.1038/srep17422. 


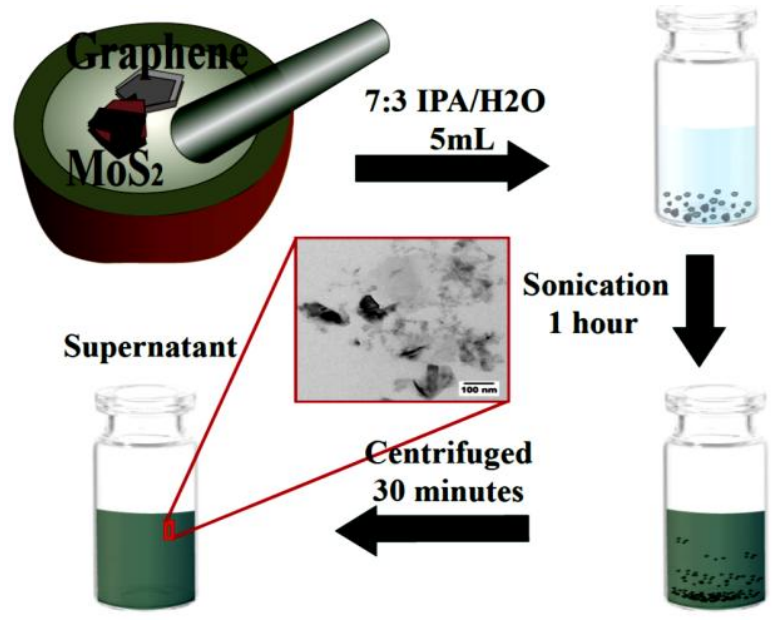

Figure-1: Synthesis of $\mathrm{MoS}_{2} /$ Graphene heterostucture as detailed in the text.
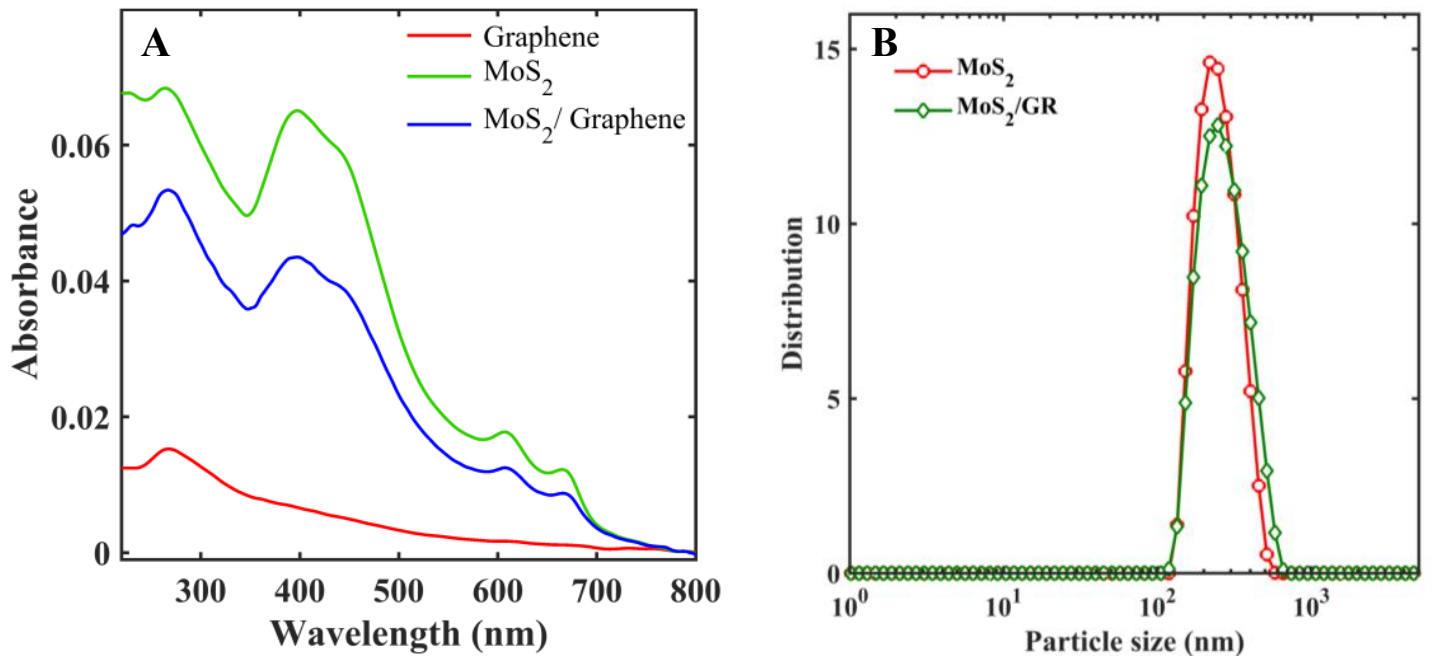

Figure-2: A) UV-Visible spectra of liquid phase exfoliated Graphene (red line), MoS (green line) and $\mathrm{MoS}_{2} /$ Graphene hetero-sturcture in 3:7 Water/Iso-propanol mixture. B) Lateral size histogram measured by Dynamic light Scattering of liquid phase exfoliated Graphene (red line), $\mathrm{MoS}_{2}$ (green line) and $\mathrm{MoS}_{2}$ /Graphene hetero-sturcture in 3:7 Water/Iso-propanol mixture. 

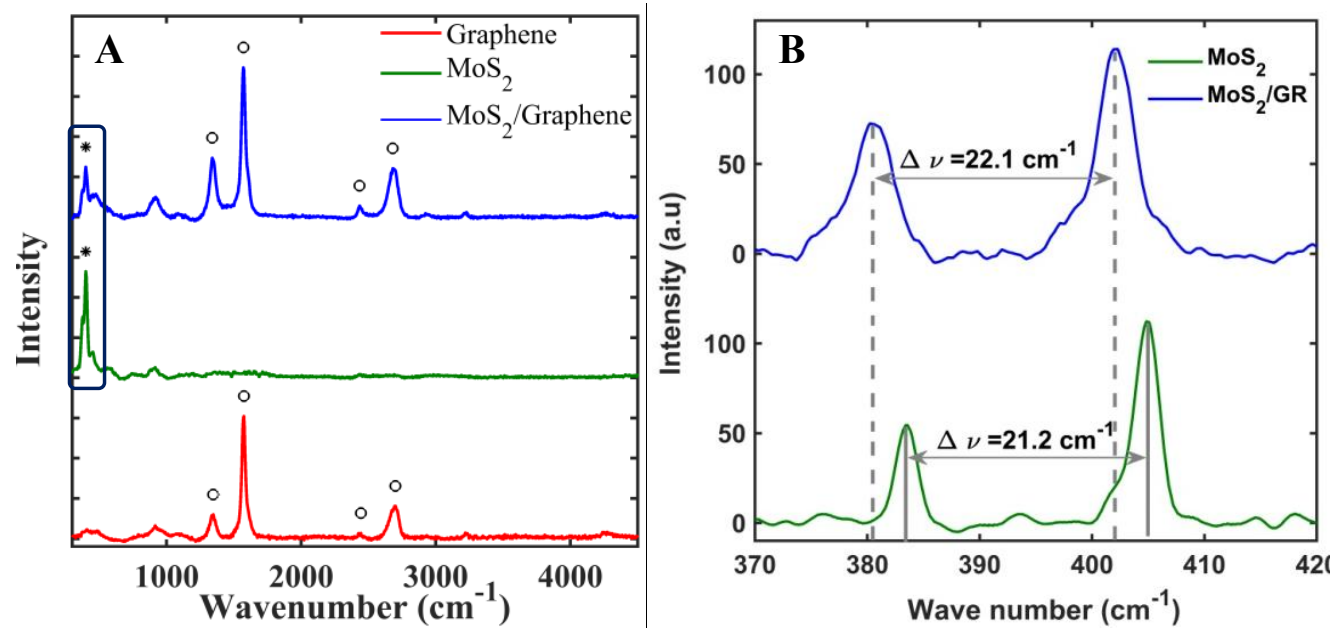

Figure-3: Micro-Raman spectra of liquid phase exfoliated Graphene (red line), $\mathrm{MoS}_{2}$ (green line) and $\mathrm{MoS}_{2} /$ Graphene hetero-sturcture in 3:7 Water/Iso-propanol mixture A) Wider scan range B) high resolution scan. 

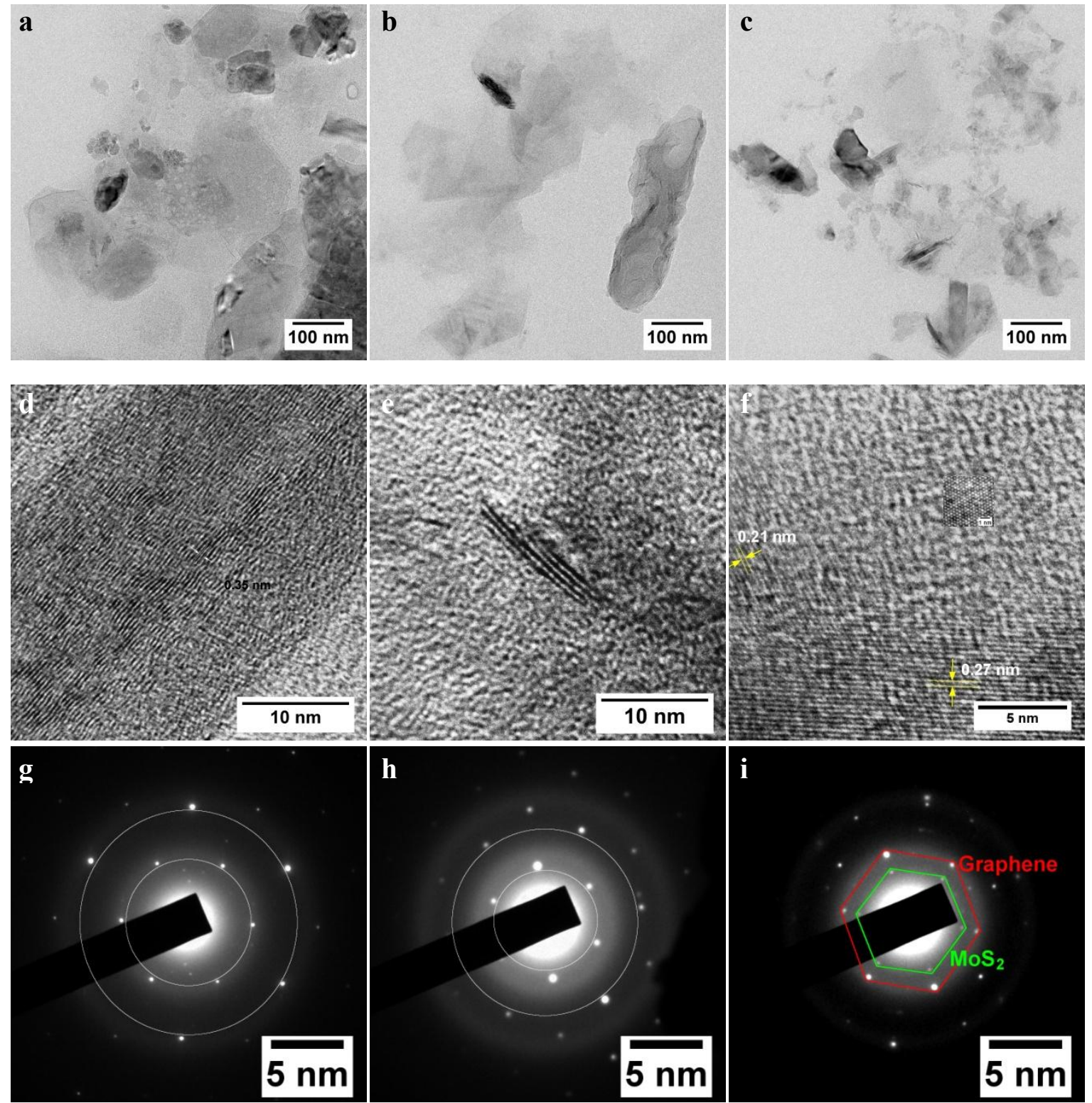

Figure-4: Transmission Electron Microscope (TEM) images: $a \& d$ Graphene Oxide; $b \& e$ $\mathrm{MoS}_{2}$ c \& $f \mathrm{MoS}_{2} /$ Graphene Oxide heterostructure. Top panel: Large area scan, Middle panel: high resolution scan. SAED pattern of $g$. Graphene Oxide; $h . \mathrm{MoS}_{2} i$. $\mathrm{MoS}_{2} /$ Graphene Oxide heterostructure 

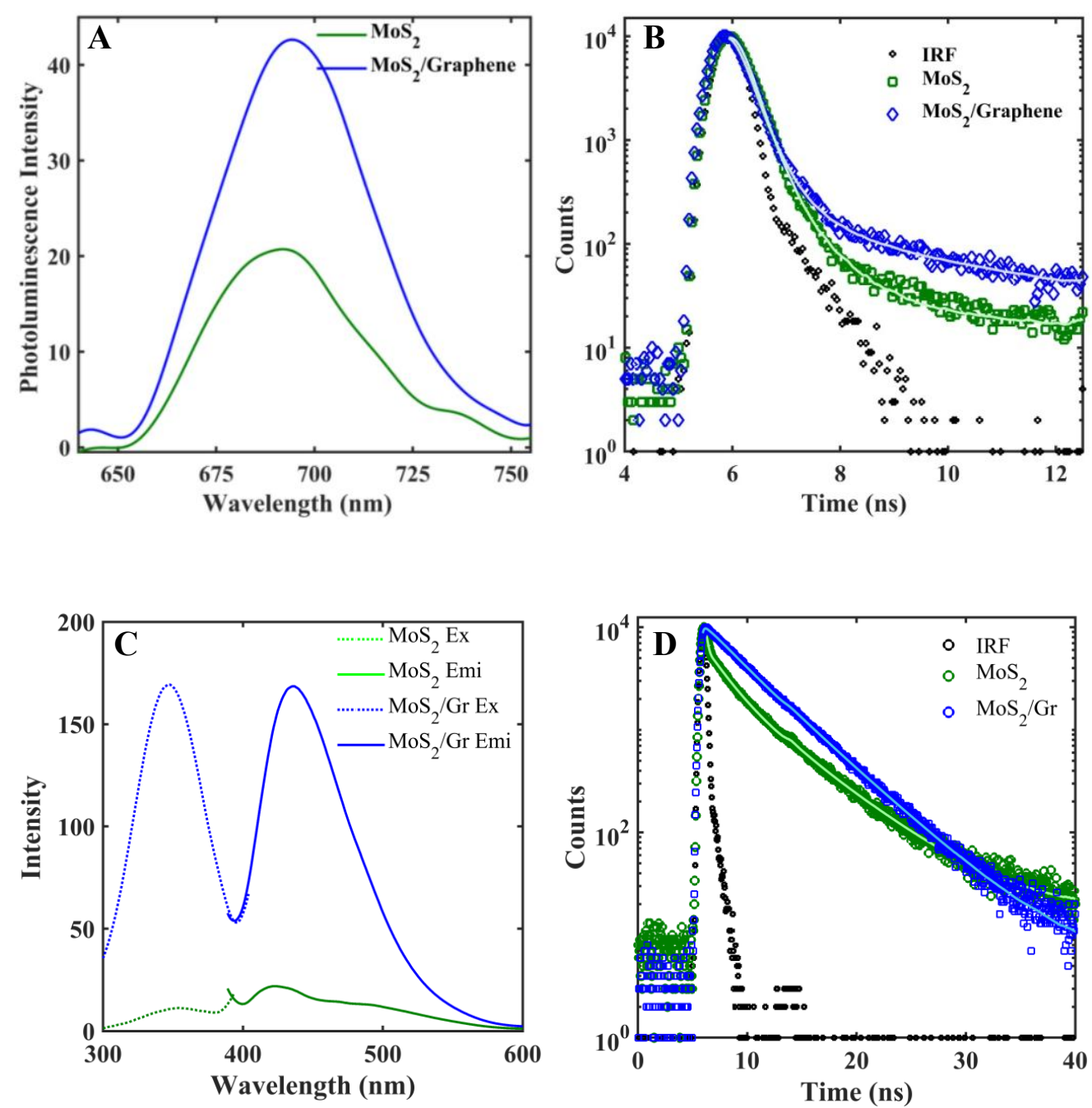

Figure-5: A) Photoluminescence excitation (dotted line) and emission spectra (solid line) of $\mathrm{MoS}_{2}$ (green line) and $\mathrm{MoS}_{2} /$ Graphene hetero-sturcture in 3:7 Water/Iso-propanol mixture; B) \& D Photoluminescence decay profile monitored at $450 \mathrm{~nm}$ and $680 \mathrm{on}$ excitation at 375 \& $470 \mathrm{~nm}$ picosecond laser diodes respectively; C) Photoluminescence emission spectra corresponding to band-gap excitation measured by exciting at $532 \mathrm{~nm}$ laser source. 


\begin{tabular}{|c|c|c|c|c|}
\hline \multirow{2}{*}{ Sample } & \multirow{2}{*}{$\lambda_{\max } \underset{(\mathrm{nm})}{\text { Emission }}$} & \multicolumn{3}{|c|}{ Decay time } \\
\hline & & $\tau_{1}(\mathrm{~ns})$ & $\tau_{2}(\mathbf{n s})$ & $<\tau>(\mathrm{ns})$ \\
\hline \multirow{2}{*}{$\mathrm{MoS}_{2}$} & $430(360)$ & $5.35(67)$ & $1.95(33)$ & 4.23 \\
\hline & $688(532)$ & $4.08(5)$ & $0.23(95)$ & 0.42 \\
\hline \multirow{2}{*}{$\mathrm{MoS}_{2} /$ Graphene } & $438(355)$ & $4.75(71)$ & $3.01(29)$ & 4.25 \\
\hline & $695(532)$ & $4.10(10)$ & $0.25(90)$ & 0.64 \\
\hline
\end{tabular}

Table-1: Photoluminescence parameters of liquid phase exfoliated $\mathrm{MoS}_{2}$ and $M o S_{2} /$ Graphene heterostructure. Excitation wavelength corresponding to the emission maximum is given in the parenthesis. Amplitude for the respective decay time was also provided in the parenthesis next to each of them. 

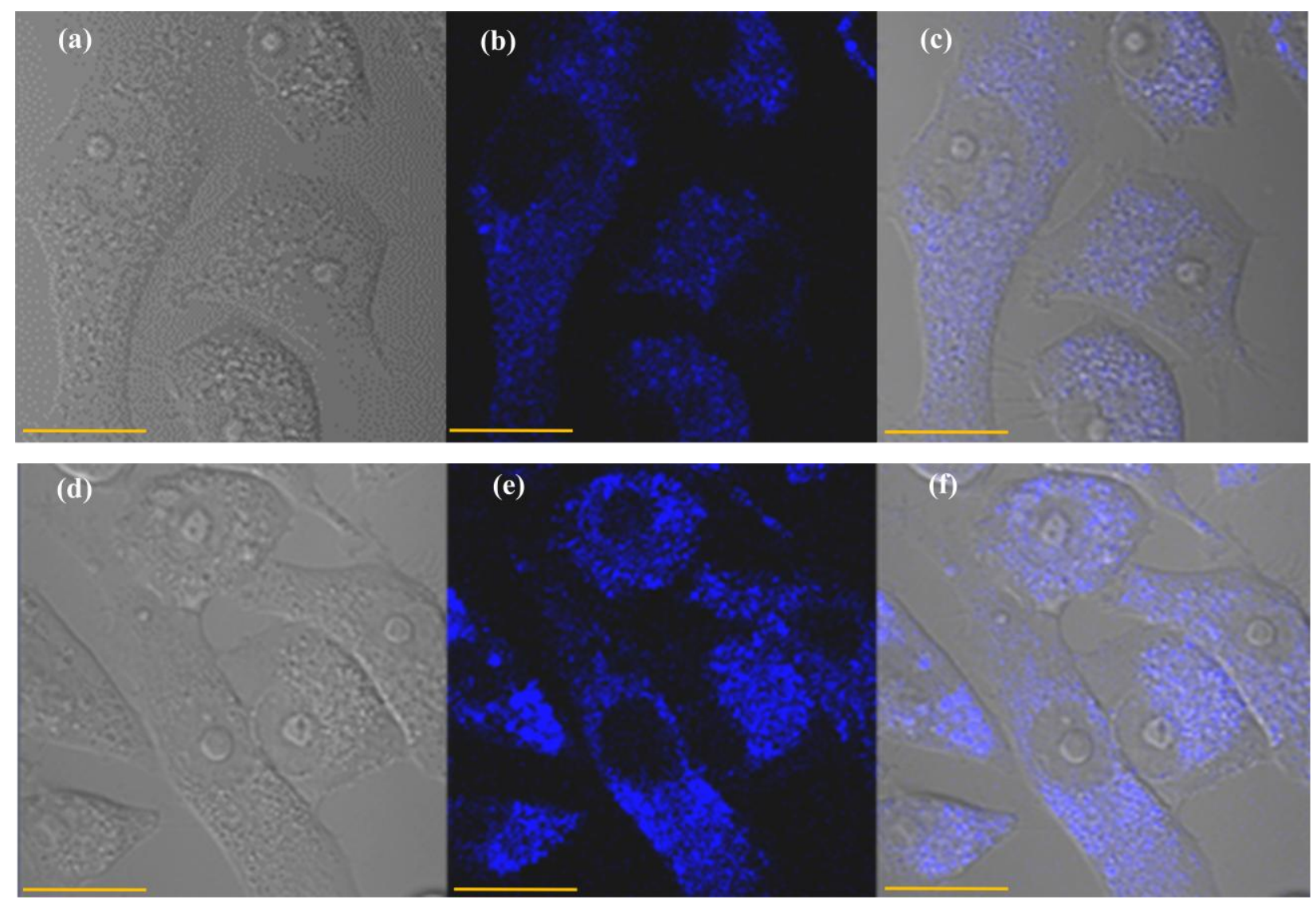

Figure-6: $a \& d$ bright field image, $b \&$ e Fluorescence image $c \& f$ merged images of Hela cells incubated for lhour $\mathrm{MoS}_{2}$ and $\mathrm{MoS}_{2}$ /Graphene hetero-structure in PBS buffer. 